GI-MS47-02

\section{Patenting polymorphs at the European Patent Office - an insider's view}

Ilaria Goss ${ }^{1}$

1. European Patent Office, Munich, Germany

email: ilariagoss@gmail.com

A number of patent applications and opposition cases in sector HBC ( Healthcare, Biotechnology and Chemistry, especially in the technical area of Heterocycles ) of the EPO relate to solid state forms of chemical compounds, in particular of active pharmaceutical ingredients. Chemical compounds can exist in a variety of solid forms including:

- amorphous solids

- polymorphs

- solvates including hydrates

- salts

- co-crystals

Different solid forms of a compound display unique physicochemical properties which can influence the manufacturability, processing, handling and intended use. For active pharmaceutical ingredients, particularly important are the thermodynamic stability and the bioavailability for poorly water-soluble drugs. Other properties may also be important including chemical stability, filterability or hygroscopicity. In practice, solid state screening seeks to find a form with an optimal balance of desirable properties. Further interest in the subject stems from the fact that solid state form inventions create intellectual property opportunities for originator and generic companies alike.

The present presentation aims at offering some guidance for the way how the EPO is dealing with applications relating to solid state inventions.

It is particularly focused on polymorphs.

Keywords: EPO, patents, legal requirements
GI-MS47-03

\section{From chemistry to crystallography or the joy of being crystallographer}

\author{
Santiago Garcia-Granda ${ }^{1}$
}

1. Department of Physical and Analytical Chemistry, University of Oviedo-CINN, Asturias, 33006, Oviedo, Spain

email:sgg@uniovi.es

Coming back to the end of my studies of chemistry, in 1980 almost 40 years ago, the crystallographic panorama was really different from what we have now. Direct Methods were really showing its real power in solving organic crystal structures and the structure of biological macromolecules were the big challenge. Powder methods were oriented to phase analysis. The development and use of Databases were very limited.

In this environment a number of young students graduates in chemistry oriented his careers to crystallography, myself among them. In my particular case, I was quite autodidact, using computer cards in the scarce facilities in Spain, and making my first crystal structures with CRYSTAN [1]. Then in my postdoc, in Nijmegen 1985-1987 with Paul Beurskens, I really experienced the flavour of a crystallography laboratory while working in the development of DirDif[2]. Since I felt myself part of the crystallographic community, of the family of the International Union of Crystallography, actively participating in meetings, committees and assemblies.

The Rietveld method [3] was a real step on the study of materials and drugs. The new synchrotron, neutron and electron sources made possible to extend the limits further than imagination. The advances in computational methods allowed to investigate more complex systems in shorter time and let crystallographic computer programs be accessible to all crystallographers or users of crystallography.

Following the personal experience and the extraordinary expansion of crystallography in the last 40 years, I shall give an overview on the opportunities for young scientist interested in crystallography and crystallographic methods.

My involvement in the IUCr activities, including the regional associations and the extraordinary impact of the International Year of Crystallography with all the activities promoting Crystallography all around the world. The IYCr legacy fund and program hopefully will revitalize the crystallographic community of the XXI century. Doubtless, the new generations of crystallographers, men and women, will continue the job of the pioneers at the beginning of the twentieth century and the expansion and splendour of Crystallography along the century to formulate the new role of Crystallography in the twenty-one century and beyond.

Acknowledgments. This work was financially supported by Spanish MINECO (MAT2016-78155-C2-1-R). 


\section{References:}

[1]. Crystan - Crystallographic Program System for Minicomputers, Bohme, R., Burzzlaf, H. and Gomm, M. Acta Cryst. Sect. A (1975) 31, S277-S277.

[2]. Logistics of a Program System - The DIRDIF Program Control and Command Protocol, Smycalla, C., Beurskens, P.T., Bosman, W.P. and Garcia-Granda, S. Journal of Applied Crystallography (1994), 27, 661-665, 4.

[3]. H. M. Rietveld, A Profile Refinement Method for Nuclear and Magnetic Structures J. Appl. Cryst. (1969). 2, 65-71.

Keywords: Chemistry, Scientific Profile, Teaching-Research

\section{GI-MS47-04}

\section{What a difference a carrier choice makes?}

Dubravka Sisak Jung ${ }^{1}$

\section{DECTRIS, Switzerland \\ email: dubravka.sisak@dectris.com}

Having this in mind that crystallography has long overcome its basic definition, it is perhaps easier to grasp that a background in crystallography offers many career choices, and that one choice does not necessarily exclude the other. In other words, background in crystallography provides you with a good set of initial parameter set (skills) that can allow a stable (career) refinement. Where exactly your career will converge depends on your refinement skills and the restraints that you (pre)define.

I have started my career with a diploma in chemistry/crystallography, and my main focus was structure determination from single crystal data. Fifteen years later I count three careers, none of them focused on the single crystal diffraction:

- research in a pharmaceutical company (crystallographer)

- basic research in crystallography (PhD student)

- business development, marketing and consultancy in a high-tech company (application scientist)

This career path features periodic alternations between academia and industry, what allowed me to extend my initial set of skills to intellectual property, teaching, good laboratory practice, X-ray instrumentation, scientific communications, marketing, spectroscopy, turbine blades for aerospace industry, cultural heritage, world airports and much more.

I am also an academic guest at the University of Zurich. I keep the International Tables of Crystallography on the desk in my study. Calculations are ongoing. 